\title{
Examining Habitability of Kepler Exoplanets
}

\author{
Maria Kalambokidis ${ }^{1}$ \\ Department of Astronomy, University of Wisconsin-Madison
}

\begin{abstract}
Since its launch in 2009, the Kepler spacecraft has confirmed the existence of 3,387 exoplanets with the goal of finding habitable environments beyond our own. The mechanism capable of stabilizing a planet's climate is the long-term carbon cycle driven by plate tectonics. We determined the likelihood of plate tectonics for Kepler exoplanets by comparing their interior structures to planets within our solar system. We used the mineral physics toolkit BurnMan to create three models with the same composition as Earth, Mars, and Mercury. We ran 19 exoplanets through the models and calculated their Mantle Radius Fraction (MRF). We found that four exoplanets may be Chthonian, four have Mercury-like MRF values, and two are Earth- like in their MRF values but did not lie within their habitable zone. In the future, as more exoplanet masses are obtained, it is likely that a greater number will appear dynamically similar to the Earth.
\end{abstract}

\section{Introduction}

Kepler Exoplanets. In the 21 st century, the field of astrobiology has grown tremendously, harnessing the technological and scientific advancements capable of addressing some of our most fundamental inquiries about life in the universe. Since its launch in 2009, the Kepler spacecraft has utilized transit photometry to explore the diversity of planetary systems beyond our own, confirming the existence of 3,387 exoplanets to date. Many studies have analyzed Kepler's confirmed exoplanets in an attempt to describe their "Earth-like" properties. Comparisons with the Earth are made considering size (radius) and orbital environment, which is described using the period, semi-major axis, and insolation flux (Batalha 2014). Most of these studies aim to describe the habitable zone for each planet — defined as the region where a rocky planet can maintain surface liquid water-by using atmospheric models looking at stellar flux distribution, planet albedo, and greenhouse gas effects (Kasting et al. 2014; Kalteneggar and Sasselov 2011; Gaidos 2013). However, additional factors should be considered when determining a planet's habitability.

Plate Tectonics. While a planet's habitable zone is a necessary factor in determining a planet's habitability, simply lying within the habitable zone does not guarantee the presence or maintenance of liquid water oceans at the surface (Foley 2015). The abundance of greenhouse gases and stellar evolution both affect the climate and maintenance of surface liquid water. Therefore, it is important to consider the mechanism capable of regulating the strength of atmospheric gases and thus countering the variations in solar luminosity: the long-term carbon cycle. By controlling atmospheric $\mathrm{CO}_{2}$ concentrations, the long-term carbon cycle stabilizes planetary climate through the temperature sensitivity of silicate weathering (Kasting et al. 1993; Berner 2004). Because of this, an active global carbon cycle is thought to be essential for maintaining liquid water on rocky

${ }^{1}$ This work was supported by a 2016 Undergraduate Research Award from the Wisconsin Space Grant Consortium. The author would like tho thank Dr. Eric Wilcots (University of Wisconsin-Madison) for his invaluable guidance, as well as UW-Madison's Department of Astronomy for providing the facilities to work on this project. 
planets in the habitable zone (Kasting and Catling 2003). In particular, plate tectonics play a vital role in the operation of the long-term carbon cycle by providing a fresh supply of surface rock for silicate weathering and driving volcanism at ridges and arcs (Foley 2015).

The driving force behind plate tectonics is the convection of the mantle. Noack et al (2014) used a two-dimensional convection model to conclude that the interior structure of a planet greatly determines plate tectonic activity and the outgassing of $\mathrm{CO}_{2}$. In 2016, Unterborn et al (2016) explored what aspects of bulk terrestrial planet composition and interior structure affect important observed properties of exoplanets, such as their mass and radius. The research group used the open-source mineral physics toolkit BurnMan to calculate mass-radius models using the Earth as a reference. The toolkit BurnMan solves equations-of-state to determine elastic properties for specified compositions in the lower mantle and can be used as a forward model on mantle composition. Through their use of BurnMan, Unterborn et al (2016), restricted their definition of "Earth-like" to planets that are dynamically similar to the Earth and thus have similar mantle radius fractions $(\mathrm{MRF}=(\mathrm{R}-$ Core $\mathrm{Radius}) / \mathrm{R})$.

In this study, we aimed to follow a similar procedure as Unterborn et al (2016) for the cataloging of the Kepler exoplanets. The goal in doing so was to determine whether we can assess the geological activity for exoplanets, despite only knowing a few basic parameters about the planets themselves. More specifically, we aimed to assess whether the interior models created for planets within our solar system — such as BurnMan — can be applied to planets beyond our solar system, such as the Kepler exoplanets. The application of these interior models is the first step in elucidating the likelihood of active plate tectonics on planets orbiting other stars, and it is thus a necessary step in determining the likelihood of their habitability.

\section{Methods}

BurnMan. The open-source mineral physics toolkit BurnMan requires the input of particular parameters about the planet: observed mass, radius, core radius, core composition, and mantle composition (Cottaar et al. 2014). In return, the program outputs a computed mass, as well as measures for both the core and mantle: the shear velocity, bulk sound velocity, density, and pressure. To begin our investigation, we used BurnMan's build_planet.py to create models for Earth, Mars, and Mercury. Our goal in doing this was two-fold, to a) check that the properties generated within BurnMan were consistent with the observed properties, and b) provide three models with varying compositions and core-mantle boundaries to run the Kepler exoplanets through. The properties used for Earth, Mars, and Mercury can be found in Table 1.

\begin{tabular}{|c|c|c|c|}
\hline & Earth & Mars & Mercury \\
\hline Mass $(\mathrm{kg})$ & $5.972 * 10^{24}$ & $6.417 * 10^{23}$ & $3.02 * 10^{23}$ \\
\hline Radius $(\mathrm{km})$ & 6,371 & 3,390 & 2,440 \\
\hline Core Radius $(\mathrm{km})$ & 3,480 & 1,700 & 2,020 \\
\hline Core & $89.9 \%$ iron & $85.4 \%$ iron & $100 \%$ iron \\
Composition & $10.1 \%$ forsterite & $14.6 \%$ stishovite & \\
\hline Mantle & $10.76 \%$ forsterite & $43.68 \%$ forsterite & $80 \%$ forsterite \\
Composition & $10.76 \%$ enstatite & $3.13 \%$ corundum & $20 \%$ enstatite \\
& $5.26 \%$ fe_perovskite & $18.7 \%$ wuestite & \\
& $25.88 \%$ periclase & $2.99 \%$ ca_perovskite & \\
& & $31.5 \%$ periclase & \\
\hline
\end{tabular}

Table 1. The masses, radiii, and internal composition for Earth, Mars, and Mercury (Fischer et al. 2016, Dreibus and Wanke 1985; Cottar et al. 2014; Unterborn et al. 2016). 
The masses generated within BurnMan for Earth, Mars, and Mercury were within 5\% of the observed values, which increased the models' validity for representing each planet. We then used the NASA Exoplanet Archive to choose potentially terrestrial Kepler planets to run through the three models (Akeson et al. 2013).

To begin, each of the exoplanets had to meet certain criterion for inclusion in our sample. First, for consistency, we only included planets detected by the Kepler Space Telescope; this narrowed the pool of confirmed exoplanets from 3,387 to 2,293. Next, we needed exoplanets whose masses have been observed. Observed masses were necessary in order to self-consistently check whether our models could reasonably be used for each exoplanet. We were able to do this by comparing the generated masses from BurnMan with the masses observed. This was a key distinction, however, because the transiting exoplanets detected by the Kepler spacecraft require follow-up radial velocity observations in order to gain measurements on their masses. Thus, there were only 229 Kepler exoplanets whose masses have been observed. Of those 229, there are 171 that have relatively constrained measurements of mass - as opposed to merely an upper limit. Finally, we needed exoplanets whose radii were less than $0.13 R_{0}\left(R_{0}=\right.$ Jupiter radius). This was necessary because; a) only rocky planets are candidates for plate tectonics, and b) BurnMan's build_planet. py is based on the Earth, and it thus only functions for planets whose radii are less than $0.13 R_{0}$. Therefore, our final sample used for this investigation was 19 Kepler exoplanets with constrained masses and radii less than $0.13 R_{0}$

Mantle Radius Fractions. We took these 19 exoplanets and ran them through the models for Earth, Mars, and Mercury. Initially, we calculated the core radius for each exoplanet by using the same core-mantle fraction $(\mathrm{CMF})$ for each respective planet (Earth $\mathrm{CMF}=.546$; Mars $\mathrm{CMF}=.501$; Mercury $\mathrm{CMF}=.828$ ). We then compared the calculated masses with the observed masses for the exoplanets in each model and adjusted the core radius accordingly in the hopes of generating a mass within $10 \%$ of the observed mass. In this way, we were able to see what interior composition produced the most reasonable masses for each exoplanet. Moreover,

we were able to then calculate the Mantle Radius Fraction (MRF) $M R F=(R-$ Core Radius $) / R$ values for each planet (Unterborn et al. 2016).

We used these MRF values to compare not only the interior composition of the exoplanet (e.g. Earth-like, Mars-like, or Mercury-like compositions) but also the interior structure. Similar to Unterborn et al (2016), we restricted our definition of "Earth-like" to those planets which are dynamically similar to the Earth and thus have similar mantle fractions. For our Earth model, we used the same MRF bounds (between 0.41 and 0.48) as Unterborn et al (2016) for defining an "Earth-like" planet. For our Mercury model, we used the same method to define the MRF "Mercury-like" bounds, which gave us a range between 0.11 and 0.21. The MRF "Mars-like" bounds were between 0.47 and 0.53 .

Habitable Zone. After elucidating which Kepler exoplanets had both a bulk composition and MRF value similar to Earth, we calculated the habitable zones for their host stars (Kasting et al.,1993). To calculate the inner and outer boundaries of the habitable zones, we first determined the

$$
M_{V}=m_{V}-5 \log \left(\frac{a}{10}\right)
$$

Where:

$m_{v}=$ apparent magnitude of the star $\mathrm{d}=$ distance from the Earth to the star (parsecs) 
absolute magnitude of the $\operatorname{star}\left(M_{v}\right)$.

Then, we calculated the bolometric magnitude of the host star $\left(M_{b o t}\right) . \quad M_{b o l}=M_{V}+B C$

The bolometric constant was determined based on the

Where: star's spectral class, using Table 2.

$\mathrm{BC}=$ bolometric correction constant

Then, using the calculated $M_{b o t}$ for the host star and observed properties of the sun, the absolute luminosity of the host star was determined:

Where:

$$
\frac{L_{\text {star }}}{L_{\text {sun }}}=10^{\wedge}\left(\frac{M_{\text {bolstar }}-M_{\text {bolsun }}}{-2.5}\right)
$$

$$
\begin{aligned}
& \frac{L_{\text {star }}}{L_{\text {sun }}}:= \begin{array}{r}
\text { absolute luminosity of the star in terms } \\
\text { of the absolute luminosity of the sun }
\end{array} \\
& M_{\text {bolstar }}=\text { bolometric magnitude of the host star } \\
& M_{\text {bolsun }}=\text { bolometric magnitude of the sun (4.72) }
\end{aligned}
$$

\begin{tabular}{|c|c|}
\hline Spectral Class & $\begin{array}{l}\text { Bolometric } \\
\text { correction constant } \\
\text { (BC) }\end{array}$ \\
\hline B & -2.0 \\
\hline A & -0.3 \\
\hline F & -0.15 \\
\hline G & -0.4 \\
\hline K & -0.8 \\
\hline M & -2.0 \\
\hline
\end{tabular}

Table 2: The general bolometric correction values for stars of spectral class B, A, F, G, K, M (Habets and Heintz 1981.

Finally, the radii of the boundaries of the host star's habitable zone was calculated for each Kepler star.

The information used to calculate the inner and outer boundaries of each host star's habitable zone was taken from the NASA Exoplanet Archive (Akeson et al. 2013).

\section{Results}

Chthonian planets. One of the first things we noticed from the results of our sample was that there were a number of exoplanets whose radii were comparable to the Earth, yet Where: $\quad r_{i}=\sqrt{\frac{L_{\text {star }}}{1.1}}$ and $r_{o}=\sqrt{\frac{L_{\text {star }}}{0.53}}$ $r_{i}=$ inner boundary of the habitable zone in astronomical units (AU)

1.1 is a constant value representing stellar flux at the inner radius (Kasting et al. 1993; Whitmire et al. 1996)

$r_{o}=$ outer boundary of the habitable zone in astronomical units (AU)

0.53 is a constant value representing stellar flux at the outer radius

(Kasting et al. 1993; Whitmire et al. 1996)

$L_{\text {star }}=$ bolometric magnitude of the sun (4.72) their masses were remarkably large. In fact, their masses were significantly greater than that of a planet made entirely of iron (Fig. 1). Indeed, even when we gave these exoplanets an interior composition of solid iron and used the lower limit of their observed mass, the masses produced in BurnMan were still less than 7\% of the observed masses. A possible explanation for these high-mass planets are that they belong to a hypothetical class of celestial objects called Chthonian planets. This term is used for a planet 
that was once a gas giant but had its hydrogen and helium atmosphere and outer layers stripped away due to close proximity to its star. If cores are massive enough, they could remain compressed with extreme densities for billions of years despite losing atmospheric mass (Hébrard et al. 2003). Upon examination, these high-mass exoplanets are indeed less than 0.5AU from their host star, providing the opportunity for Chthonian formation.

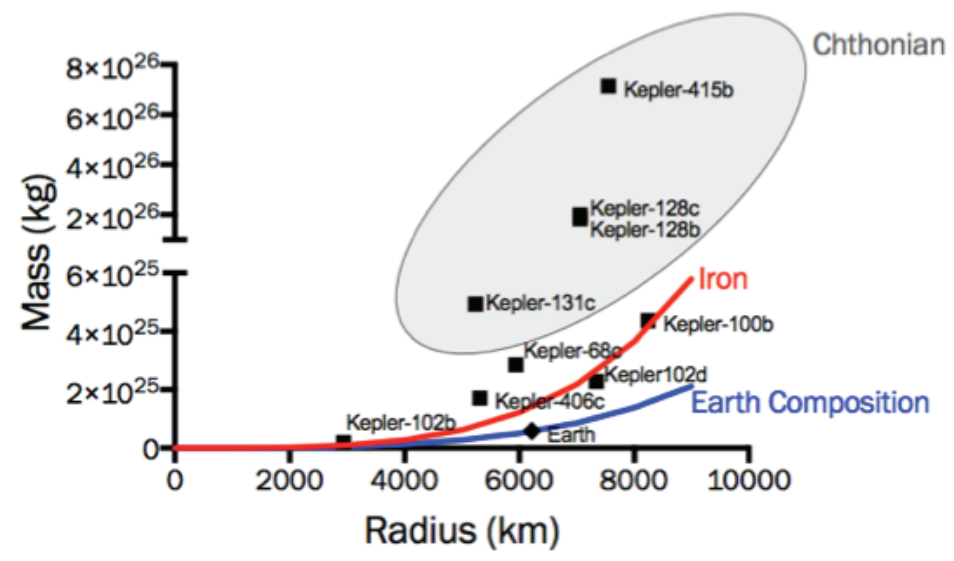

Fig. 1. A plot of the mass $(\mathrm{kg})$ and radius $(\mathrm{km})$ for the exoplants in our sample whose masses could not be retrieved using an Earth, Mars, or Mercury composition. A blue line indicating the masses and radii of planets with an Earth-like composition, as well as a red line indicating the masses and radii of planets made entirely of iron, are shown for reference. Earth is shown for reference ( $)$. Four possible Chthonian planets are circled in gray (Kepler-131c, mass: $4.9348 \mathrm{e}+0.25$; Kepler-128c, mass: $1.81406 \mathrm{e}+025$; Kepler-128c, mass: $1.9929 \mathrm{e}+026$, Kepler-415b, mass: 7.15546e-026).

Earth-like. There were seven exoplanets whose masses were retrieved using our model with an Earth-like composition. Of these seven, two exoplanets had MRF values that fell within the Earthlike MRF bounds; Kepler-102f $(\mathrm{MRF}=0.45)$ and Kepler-78b $(\mathrm{MRF}=0.43)$. The results are shown in (Fig. 2).

\section{Earth-like Composition}

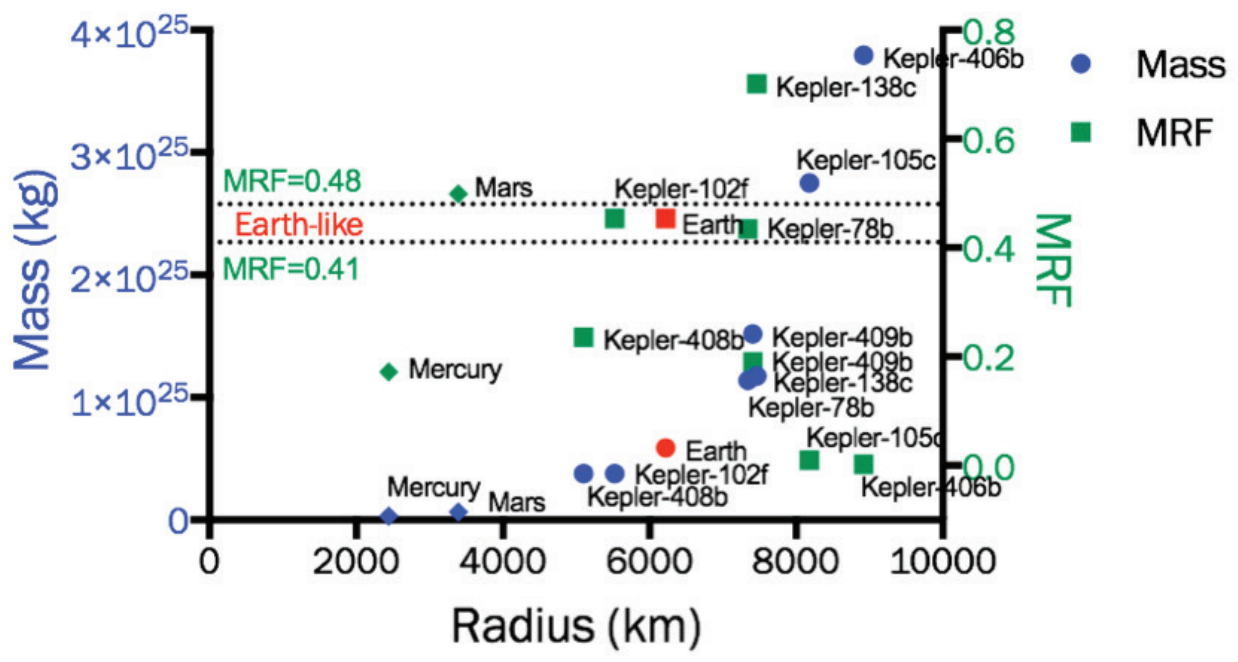

Fig. 2: A plot of mass $(\mathrm{kg})$ in blue on the left y-axis, MRF in green on the right y-axis, and radius $(\mathrm{km})$ on the $\mathrm{x}$-axis are shown for the seven exoplanets whose masses were retrieved using an Earth-like composition. Earth is shown in red, as well as Mercury and Mars $(\diamond)$ for reference. The Earth-like MRF bounds $(0.41$ and 0.48 ) are indicated as dotted lines. 
Mercury-like. There were no exoplanets whose observed masses were retrieved using a Mars-like composition that were not already generated using an Earth-like composition. Additionally, none of them had Mars-like MRF values. For these reasons, we did not include the results of our Mars model in our analyses. There were, however, nine planets with observed masses aligning with those produced within BurnMan when we used a Mercury-like composition. Of these nine, four of them had MRF values that fell within the Mercury-like range (from 0.11 to 0.21 ): Kepler-408b $(\mathrm{MRF}=0.24)$, Kepler-409b $(\mathrm{MRF}=0.23)$, Kepler-105c $(\mathrm{MRF}=0.17)$, and Kepler-406b $(\mathrm{MRF}=0.17)$. The results are shown in (Fig. 3).

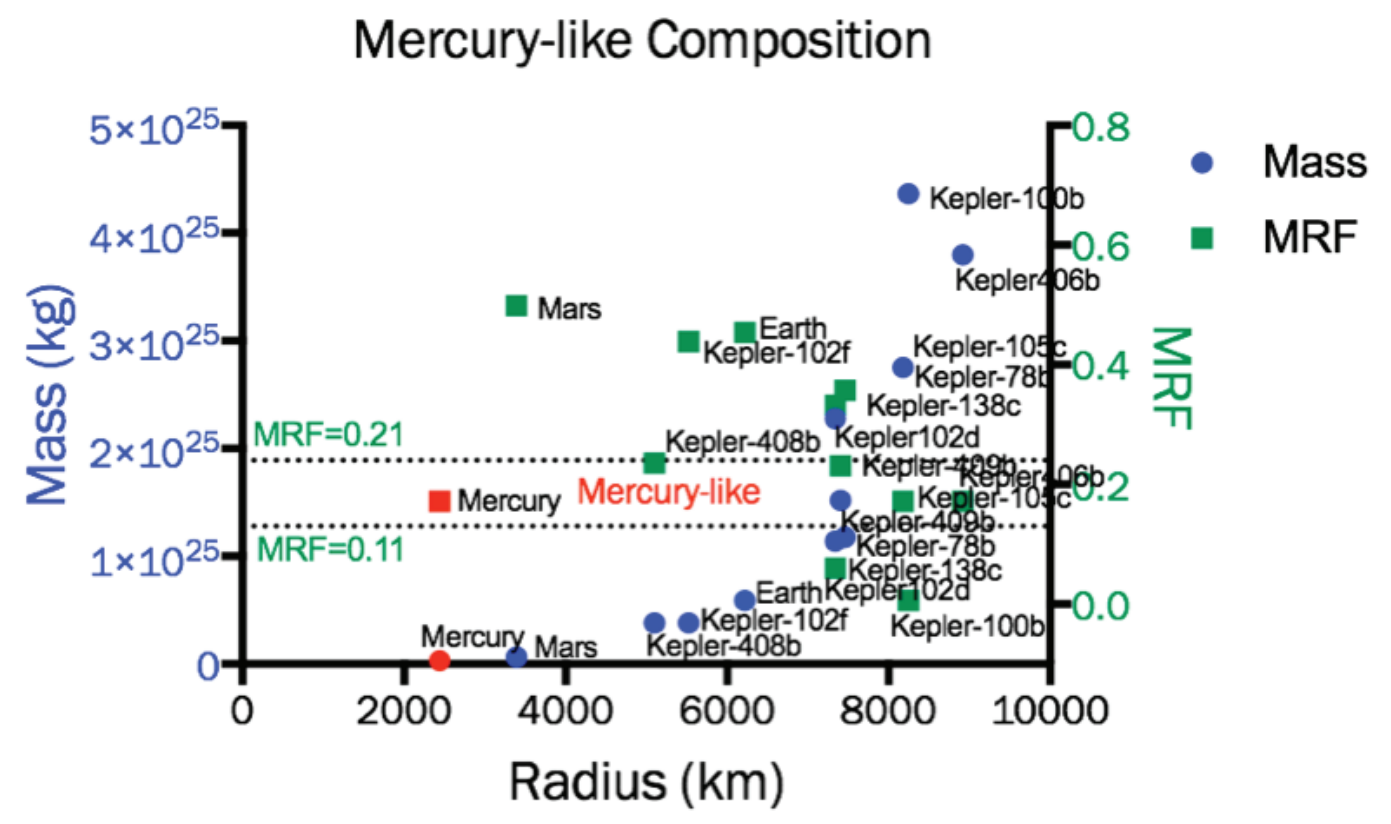

Fig. 3: A plot of mass $(\mathrm{kg})$ in blue on the left y-axis, MRF in green on the right y-axis, and radius $(\mathrm{km})$ on the $\mathrm{x}$ - axis is shown for the nine exoplanets whose masses were retrieved using a Mercury-like composition. Mercury is shown in red, as well as Earth and Mars for reference. The Mercury-like MRF bounds (0.11 and $0.21)$ are indicated as dotted lines.

Habitable Zones. The last phase was to calculate the habitable zones for the two exoplanets whose masses, radii, MRF values, and bulk compositions were comparable to the Earth's: Kepler-102f and Kepler-78b. Additionally, their shear and bulk sound velocities of the mantle were similar to the Earth's, as seen in Table 3. Thus, it is likely that these two planets are dynamically comparable to the Earth and are therefore potential candidates for active plate tectonics (Unterborn et al. 2016).

\begin{tabular}{|l|c|c|}
\hline & Shear Velocity $(\mathrm{km} / \mathrm{s})$ & Bulk Sound Velocity $(\mathrm{km} / \mathrm{s})$ \\
\hline Earth & 6.21 & 11.18 \\
\hline Kepler-78b & 6.48 & 12.4 \\
\hline Kepler-102f & 5.98 & 9.93 \\
\hline
\end{tabular}

Tabe 3. The shear velocity and bulk sound velocity for Earth, Kepler-78b, and Kepler-102f.

However, Kepler-78b (Semi-major axis: 0.009AU) did not lie within its habitable zone $\left(r_{i}=0.605 \mathrm{AU} ; r_{o}=0.872\right)$, nor did Kepler-102f (Semi-major axis: $0.165 \mathrm{AU} ; r_{i}=0.165 \mathrm{AU}$; $\left.r_{o}=0.727 \mathrm{AU}\right)$. 


\section{Conclusion}

Through this investigation, we have been able to draw a few key conclusions. First, we learned that a model created based on the Earth's interior properties (BurnMan) is not only consistent for well-known planets (Mercury and Mars) but also for planets for whom we only know a few basic properties (e.g. mass, radius). The reasonable numbers generated within BurnMan for these exoplanets supports the idea that the minerals that are common within our solar system are likely common in other planetary systems. These interior models, therefore, may be a powerful tool in the future for assessing geological activity on planets beyond our solar system.

Secondly, it appears that exoplanets with internal structures similar to Earth and Mercury are not uncommon. Because we are still in the early stages of radial velocity measurements - and we need estimates on exoplanet masses - our sample was only 19. This is important to note for a number of reasons:

1) It is easier to detect high-mass objects orbiting their host star using the radial velocity method than it is to detect low-mass planets akin to Earth or Mercury. Thus, it is unsurprising that possible Chthonian planets appeared in our sample, as they would be the easiest to detect after filtering out planets with large radii.

2) It is easier to detect planets with small semi-major axes using the radial velocity method than it is to observe planets farther away - and possibly within the habitable zone. Thus, it is unsurprising that the exoplanets within our sample were very close to their host stars, including those that appeared to have similar bulk compositions and structures as the Earth.

3) As a scientific community, we have only just begun to obtain mass measurements for the confirmed Kepler exoplanets. Therefore, it is reasonable that a greater portion of our sample appeared to be Mercury-like than like Earth or Mars; they were all very close to their host star, just as Mercury is near the sun.

As we gain more estimates on the masses of exoplanets, it is then likely that we will obtain measurements on planets farther away from their host stars. Because the internal structure of planets with small semi-major axes appeared Mercury-like, we may predict that those farther away from their star may appear more like Earth and Mars in their interior structure. It is possible then that these are the planets that lie within the habitable zone.

We won't have to wait long for these radial velocity measurements. In the next decade, there are significant projects planned to make these observations, including NASA-NSF Exoplanet Observational Research (NN-Explore) in 2019 and the Wide-Field Infrared Survey Telescope (WFIRST) which will launch in the mid-2020s. As we learn more about planetary systems beyond our own, we can continue to assess their likelihood for plate tectonic activity using interior models for planets within our solar system. In doing so, we will move forward in assessing their potential to sustain a hospitable climate and perhaps harbor life. 


\section{References}

Akeson, R. L., Chen, X., Ciardi, D., Crane, M., Good, J., Harbut, M., Jackson E., Kane S.R., Laity A.C., Leifer S., Lynn, M. (2013). The NASA exoplanet archive: data and tools for exoplanet research. Publications of the Astronomical Society of the Pacific, 125(930), 989.

Batalha, N. M. (2014). Exploring exoplanet populations with NASA's Kepler Mission. Proceedings of the National Academy of Sciences, 111(35), 12647-12654.

Berner, R. A. (2004). A model for calcium, magnesium and sulfate in seawater over Phanerozoic time. American Journal of Science, 304(5), 438-453.

Cottaar, S., Heister, T., Rose, I., \& Unterborn, C. (2014). BurnMan: A lower mantle mineral physics toolkit. Geochemistry, Geophysics, Geosystems, 15(4), 1164-1179.

Dreibus, G., \& Wanke, H. (1985). Mars, a volatile-rich planet. Meteoritics, 20, 367-381.

Fischer, R., Campbell, A., \& Ciesla, F. (2016, April). The composition of Earth's core from equations of state, metal-silicate partitioning, and core formation modeling. In EGU General Assembly Conference Abstracts (Vol. 18, p. 8417).

Foley, B. J. (2015). The role of plate tectonic-coupling and exposed land area in the development of habitable climates on rocky planets. The Astrophysical Journal, 812(1), 36.

Gaidos, E. (2013). Candidate planets in the habitable zones of Kepler stars. The Astrophysical Journal, 770(2), 90.

Habets, G. M. H. J., \& Heintze, J. R. W. (1981). Empirical bolometric corrections for the main- sequence. Astronomy and Astrophysics Supplement Series, 46, 193-237.

Hébrard, G., Étangs, A., Vidal-Madjar, A., Désert, J. M., \& Ferlet, R. (2003). Evaporation rate of hot Jupiters and formation of Chthonian planets. arXiv preprint astro-ph/0312384.

Kaltenegger, L., \& Sasselov, D. (2011). Exploring the habitable zone for Kepler planetary candidates. The Astrophysical Journal Letters, 736(2), L25.

Kasting, J. F., \& Catling, D. (2003). Evolution of a habitable planet. Annual Review of Astronomy and Astrophysics, 41(1), 429-463.

Kasting, J. F., Kopparapu, R., Ramirez, R. M., \& Harman, C. E. (2014). Remote life-detection criteria, habitable zone boundaries, and the frequency of Earth-like planets around M and late K stars. Proceedings of the National Academy of Sciences, $111(35), 12641-12646$.

Kasting, J. F., Whitmire, D. P., \& Reynolds, R. T. (1993). Habitable zones around main sequence stars. Icarus, 101(1), $108-128$.

Noack, L., Godolt, M., von Paris, P., Plesa, A. C., Stracke, B., Breuer, D., \& Rauer, H. (2014). Can the interior structure influence the habitability of a rocky planet?. Planetary and Space Science, 98, 14-29.

Unterborn, C. T., Dismukes, E. E., \& Panero, W. R. (2016). Scaling the Earth: A Sensitivity Analysis of Terrestrial Exoplanetary Interior Models. arXiv preprint arXiv:1510.07582.

Whitmire, D. P., \& Reynolds, R. T. (1996). Circumstellar habitable zones: astronomical considerations. In L. R. Doyle (Ed.), Circumstellar habitable zones (Vol. 1, p. 117). 